\title{
CRIPTOS ACTIVO PARA LA INTERMEDIACIÓN E INVERSIÓN FINANCIERA EN COLOMBIA
}

\section{CRYPTOS ACTIVE FOR INTERMEDIATION AND FINANCIAL INVESTMENT IN COLOMBIA.}

\section{José Alfonso Mendoza Gallego ${ }^{1}$ \\ Francisco Rivelino Bernal Cerquera ${ }^{2}$}

Grupo de investigación

ESINSUR

El presente artículo de investigación pretende determinar cómo los Criptoactivos que participan en la intermediación financiera, captan el dinero que hace una persona de forma individual o jurídica, el intermediario financiero se refiere a la recaudación de ingresos de estas

\section{José Alfonso Mendoza Gallego}

Doctorando en Dirección y Finanzas, Magister en Educación de la Diversidad, Administrador Bancario y Financiero, Tecnólogo en Gestión Comercial y Negocios, Docente Investigador Asociado (I) Min ciencias, Grupo de investigación ESINSUR de la Facultad de Economía y Administración, de la Universidad Sur colombiana de Neiva - Colombia ORCID 0000-0002-0002-4104. Correo: jose.mendoza@usco.edu. $\underline{\mathrm{co}}$

\section{$2 \quad$ Francisco Rivelino Bernal Cerquera}

Doctorando en Pensamiento Complejo de Multiversidad Mundo Real (México) Administrador Financiero, Especialista en Alta Gerencia, Magíster en Educación, Magíster MBA en Administrador de Empresas, Docente Investigador Junior Min ciencias, grupo ESINSUR de la Facultad de Economía y Administración, de la Universidad Sur colombiana de Neiva - Colombia, https://orcid.org/0000-003-2976-1033. francisco.bernal@usco.edu.co personas, que luego se distribuyen en forma de préstamos y fondos mutuos. De ese modo devengar intereses o cualquier otro beneficio propicio para quienes poseen fondos, $o$ en beneficio para los intermediarios.

Después del año 2020, lleno de incertidumbre, el año 2021 se presenta como año de recuperación financiera, en la que una gran cantidad de liquidez está lista para invertir en múltiples activos. Y ahora es más que nunca, es el momento de hablar de indumentarias o herramientas como una alternativa real de un sistema financiero centralizado y como un buen refugio para los próximos años.

La digitalización y apertura de los mercados actuales y la capacidad máxima de 
las operaciones que se realizan actualmente, además de acercarse a una definición que permite la garantía de la intermediación e inversión financiera en Colombia al explicar las formas de que la administración de intervención en la economía tiene cómo lidiar con nuevas perspectivas que se adaptan a la digitalización de los mercados e identifican cada uno de los elementos que constituyen la obligación tributaria en la intervención general del estado el mercado de Criptoactivos.

\section{PALABRA CLAVE}

Los Criptoactivos, Intermediación, Inversión, Financiera, Colombia

\section{ABSTRACT}

This research article aims to determine how Cryptoassets participate in financial intermediation, which is the raising of money made by an individual or legal person, the financial intermediary refers to the collection of income from people, which are then distributed in the form of loans and mutual funds. In this way, accrue interest or any other favorable benefit for those who have funds, or for the benefit of intermediaries.

After 2020, full of uncertainty, 2021 is presented as a year of financial recovery, in which a large amount of liquidity is ready to invest in multiple assets. And now is more than ever, the time when more to talk about clothing or tools as a real alternative to a centralized financial system and as a good refuge for the next few years.

The digitization and opening of current markets and the maximum capacity of the operations that are currently carried out, in addition to approaching a definition that allows the guarantee of financial intermediation and investment in Colombia by explaining the ways in which the administration intervenes in the The economy has how to deal with new perspectives that adapt to the digitalization of markets and identify each of the elements that constitute the tax obligation in the general intervention of the state in the Cryptoassets market.

\section{KEYWORDS:}

Crypto Assets, Intermediation, Investment, Financial, Colombia

\section{INTRODUCCIÓN}

En los últimos años, se han llevado a cabo varias encuestas sobre este tema, para contribuir a las personas que necesitan invertir y encontrar ventajas en el momento de la demanda. Se refiere al estudio de Criptoactivos como una inversión alternativa en Colombia, análisis operacional, riesgos, su estatus de regulación actual, teniendo en cuenta la posición del Banco de la República y la Superintendencia Financiera, y su posibilidad de usarse o hacerse efectiva.

En ese mismo orden de idea para (Moreno at al 2018) presenta un inversor a una gran cartera de opciones para decidir obtener devoluciones, de altura o baja, dependiendo del grado de riesgo que le ofrezca a su dinero que está dispuesto a hacerse cargo. El banco comercial, por ejemplo, ofrece varias de estas opciones, que son ingresos fijos o variables, como fondos de inversión colectiva, aunque con bajas tasas de rentabilidad, son ideales para inversores conservadores, a través de quienes operaran los Criptoactivos, que para (Hurtado 2021) las criptodivisas o criptomonedas son una moneda digital o virtual diseñada para funcionar como un medio de intercambio. Para garantizar transacciones o envío de monedas, se utiliza el cifrado, así como controlar la creación de nuevas unidades de una criptomonía en particular, las personas también pueden encontrar acciones o 
bonos de valor, inversiones que tienen un cierto nivel de riesgo, cantidad mínima de inversión y límite de tiempo para el retorno, todos estos factores importantes son para analizar antes de decidir dónde invertir en ellos.

Un sector de inversión con alta volatilidad o riesgo, tanto para obtener cuánto se pierde, en la negociación de divisas que consiste precisamente en la compra y la venta de criptomonedas, estos a través de una plataforma administrada por el corredor (agente o intermediario), donde se deposita la inversión. Se opera directamente en corto o largo plazo

Es por ello que un inversionista tiene una amplia cartera de opciones en las que decide obtener devoluciones, ser altas o bajas, dependiendo del grado de riesgo de que el que le dé su dinero esté dispuesto a asumir. La banca comercial, por ejemplo, ofrece varias opciones de este tipo, es un ingreso fijo o variable, como los fondos de inversión colectiva que, aunque la baja rentabilidad, son ideales para los inversores conservadores. Gracias a los comisionados de stock, las personas también pueden encontrar acciones o valiosos, inversiones con un cierto nivel de riesgo, una cantidad mínima de inversión y un tiempo de espera para el retorno, todos estos factores importantes que se analizarán antes de decidir invertir.

En ese mismo orden de idea Chirino (2021) señala que el aumento de las transacciones en los diferentes criptoactivos, llamaba a los diferentes de gobiernos., que no están interesados en las transacciones generadas por Criptoactivos, pero por temor a que se usen para la legitimación de capital y el financiamiento terrorista, porque se entiende que las operaciones realizadas en Bitcoin estaban bajo la forma de anonimato absoluto, lo que permite la elusión de un sistema que permite pagos seguros en línea con el nombre de "tokens" virtuales, que están representados por las entradas contables internas del sistema

No obstante, uno de los mayores desafíos presentado por el mercado actual es acordado mediante una definición unitaria de criptoactivos y se han inventado términos criptoactivos y se han inventado los legisladores y reguladores sin discriminación, como la corriente, la criptomoneda, las criptodivisas, la moneda virtual se normalizan digitalizada divisa.

Para esta investigación el propósito es determinar cómo los Criptoactivos que participan en la intermediación financiera, que es la captación de dinero que hace una persona individual o jurídica en Colombia. En medio de todas estas alternativas invertidas, ha surgido una nueva forma de atraer la rentabilidad: transacciones con monedas virtuales o Criptoactivos. En este artículo se va a determinar ¿Cuáles son los Criptoactivo? ¿en qué principios se basan y cuáles son los más importantes para la capitalización del mercado en Colombia? En sus inicios, los criptones atrajeron el aspecto de muchas personas por su perspectiva de la rentabilidad y por el pequeño esfuerzo que tuvo que llevar a cabo su transacción. Luego, el enfoque cambia, ya que la criptactiva "adquiere" algunas características del dinero, estas operaciones no se llevaron a cabo dentro del esquema tradicional del mercado financiero, por el contrario, fueron operaciones inmediatas entre los usuarios, sin la necesidad de la intermediación financiera tradicional, Las respectivas Haciendas que no recibieron ninguna contribución para este tipo de operación. Esta alternativa virtual puede incluso ser almacenada como una inversión en el largo tiempo, ya que su modo de operación es similar a la de las monedas (incluso si no tiene un beneficio realmente fijo), la plataforma 
de bloques es la herramienta más utilizada para su transacción siendo la que proporciona una mayor seguridad informática.

\section{MARCO TEÓRICO.}

\section{CRIPTOS ACTIVO.}

Para Hurtado (2021) las criptomonedas son monedas digitales o virtuales que funcionan como un soporte de reemplazo. Para garantizar transacciones o enviar monedas, utiliza criptografía y control de nuevas unidades de criptomona especial (p.2). En resumen, las criptomonadas son entradas limitadas en una base de datos en la que nadie puede cambiar a menos que se cumplan las condiciones especiales.

Sus principales características i

- Una Criptomoneda o Criptodivisa es un formulario de activos digitales basado en una red distribuida en una gran cantidad de computadoras. Esto lo hace descentralizado (no depende de una computadora central grande).

- Esto no es una moneda física (aunque se puede almacenar en dispositivos como discos duros o una memoria externa).

- Su creación se basa en la criptografía única.

- No dependen de una agencia o gubernamental responsable de producir este tipo de moneda.

En ese mismo orden de idea para (Chirinos 2020), uno de los mayores desafíos presentado por el mercado actual, es acordado por una unidad de función criptoactiva, que ha inventado diferentes términos que los legisladores y las autoridades regulatorias donde se utilizan sin discriminación, como Curriplon, Cryptomon, Cryptodivis, Moneda Virtual para ser la sala digital de uso normalizado.
Una definición basada en las características de la criptoactiva, en su forma más pura, se puede definir como una persona hecha en forma digital sin la necesidad de ir a la autoridad central, utilizando códigos matemáticos en el evolutivo cluscbytrográfico para aprobar la confirmación de las transacciones realizadas por los usuarios. Así que también bajo los esquemas cifrados, se puede garantizar el anonimato parcial.

Para el (BCSM 2021) La primera moneda virtual totalmente implementada es el Bitcoin. Esta moneda virtual entró en funcionamiento en el año 2009 y, con el tiempo, han ido apareciendo diferentes monedas virtuales como Litecoin (LTC) (2011), Ripple (XRP) (2012) o Ethereum (2015). Tether (USDT)

\section{INTERMEDIACIÓN FINANCIERA.}

Para Chacón (2017) Al describir el sistema financiero, siempre es una referencia a su propósito principal, es la intermediación que recibe dinero de los ahorradores por medio de la formalización de los depósitos, que luego se canalizan a quienes necesitan financiamiento, a través de operaciones de préstamo, crédito o pago anticipado.

El procedimiento interino financiero es conocido, al menos, de la Edad Media alta. Los comerciantes solían aceptar depósitos de clientes, proveedores o ciudadanos simples que entendían que su dinero era más seguro en los almacenes protegidos y tutorías de los ricos comerciantes que en su propia casa. Para este servicio de guardia, lógicamente, el precio fue acusado. Pero en algún momento, los comerciantes entendieron que podían prestar el dinero que depositaban en sus cofres cada vez que lo arreglaron cuando sus dueños legales lo exijan. Debido a que estaban al tanto del tema realizado por sus finanzas o por cualquier otra 
razón, estos comenzaron a disparar. De esta manera, el comerciante que se convirtió en un banquero, pagado, quien proporcionó fondos.

Para (García at al 2011) La información que generan los intermediarios financieros es utilizada en dos tipos de aplicaciones: (1) para relacionar transacciones financieras, asociando recursos y necesidades, entre diversos agentes, y (2) para gestionar los riesgos y transformar la naturaleza de las demandas financieras, como cuando un banco produce información de carácter crediticia para controlar el riesgo de crédito de un prestatario. En el primer caso la información que generan los intermediarios financieros actúa como brokers, y en el segundo caso, como transformadores de las cualidades financieras de los activos. Estas aplicaciones son parte fundamental de la intermediación financiera ( $p$ 108).

Por ello cuando los intermediarios financieros actúan como brokers, además de relacionar, entre sí, las demandas y ofertas financieras de diferentes agentes económicos, también analizan e interpretan información, en busca de señales que le permitan tomar una buena decisión, en cuanto a comprar, vender o mantener su posición en productos del mercado financiero, o en cuanto a seleccionar un agente determinado en el cual invertir, en el caso de otorgamiento de créditos y financiamiento de proyectos; contribuyendo de esta manera en la solución del problema de asimetría de la información. Esta función les permite ser intermediarios financieros, desarrollar habilidades en el análisis de información, que no poseen demandantes y oferentes.

Así mismo el papel de los intermediarios financieros, que es contribuir a la solución de la selección hostil y la duplicación del examen se vuelve más importante, cuando el objeto de un estudio no es fácilmente visible, un hecho que obliga a los intermediarios financieros a mejorar la capacidad de observar la información, la especificidad inactivada. y. Instrucción, y este nivel de capacidad, es sobre aquellos que leen su competencia.

El riesgo moral es un problema posterior al control que se caracteriza porque:

(1) el nivel de esfuerzo para el agente (una de las partes en un Contrato) no es un cable verificable, un hecho que puede afectar el resultado financiero de una operación en el caso de que el agente no esté interesado en conservar los intereses del director (el otro del contrato de las partes, que en nuestro estudio está representado por los intermediarios financieros o (2) el nivel de esfuerzo del agente es un cable verificativo, pero esto con información privada, después del contrato que se puede usar dañando el interés de la principal; En ambos casos, la causa puede responder al hecho de que los intereses diferirán en gran medida de los del director, por lo que, por lo tanto, un bajo nivel de esfuerzo, o la información privada, del agente, no fallaría su nivel de utilidad, pero lo haré. Influencia de él desde la principal, generando asimetría en la información. Por lo tanto, es la tarea de las pérdidas que mitigan las IFS, un producto de riesgo moral, de estrategias de monitoreo, como el seguimiento de los compromisos contractuales; (tales como liquidez, duración, riesgo, divisibilidad), mayores de contratos ajustables, donde los agentes privan al estímulo a eficientes, transformadores de las cualidades o atributos financieros de los activos.

Cuando los intermediarios financieros actúan como transformadores de las cualidades finales o atributos finales (como la liquidez, la duración, el riesgo, la divisibilidad), sus objetivos principales son los de generación de economías de escala en las operaciones financieras, 
diversifican los riesgos y permiten un grado de ajuste, monedas y fechas límite, adecuadas entre los activos y pasivos.

\section{INVERSIÓN FINANCIERA.}

Para (Lorenzo 2017), la estructura del Sistema Financiero Colombiano es un sistema financiero, un conjunto de varias instituciones que realizan operaciones para transferir recursos de un sector excedente (depósito o inversión) para un sector de déficit (compañías que requieren financiar sus proyectos); El sistema financiero comprende el mercado bancario o el mercado intermedio y el mercado de valores o des intermediario. Por lo tanto, el sistema financiero desempeña un papel principal en la economía porque funciona como medios de gestión de riesgos, proporciona un sistema de pago y permite la asignación de un precio financiero. ( $p$ 10)

Así mismo en la inversión financiera es esencial la gestión de riesgos que consiste en minimizar el riesgo de incumplimiento cuando existe una asimetría de información entre los subcontratistas en los contratos, ya que el acreedor no sabe o no puede conocer todas las condiciones económicas del deudor.

Es por ello que, en el mercado intermedio, los bancos capturan los recursos de los profesionales y evalúan a los posibles deudores para realizar estos recursos bajo su responsabilidad, es decir, el solicitante no tiene el riesgo del deudor del Banco, su riesgo ya es el Banco. Intermediación, que cubre sus costos y gastos.

No obstante, en el mercado de valores, los inversores pueden reducir el riesgo de invertir en valores a través de la provisión de sus recursos o valores a un intermediario del mercado de valores, ya que tiene conocimiento sobre las condiciones económicas de los emisores de valores.

Los sistemas de pago son los mecanismos para transferir recursos a lo largo del tiempo y el espacio sin demanda de envíos, como revoluciones, transferencias de dinero en el mercado bancario y valores.

La provisión de un sistema de pago ofrece una mayor certeza del conocimiento de los pagos y los publicados sobre los pagos de la posibilidad de que involucre el agente de software de hardware y generalmente le brinda a la economía una mayor liquidez, porque constituyen el pago de obligaciones.

En ese orden de idea (Hurtado 2021), en medio de todas estas alternativas de inversión, surgió una nueva manera de atraer rentabilidad: las transacciones con monedas virtuales 0 Criptomonedas para determinar cómo los financiera que es la captación de dinero que hace una persona individual o jurídica. En sus inicios, las Criptomonedas atrajeron la mirada de muchas personas por su perspectiva de rentabilidad y por el poco esfuerzo que se requería para llevar a cabo su transacción. Esta alternativa virtual, incluso se puede mantener como una inversión en el largo tiempo toda vez que su modo operante es similar al de las divisas (a pesar de que no tiene una rentabilidad realmente fija), siendo la plataforma Blockchain la herramienta más utilizada para su transacción por ser la que brinda mayor seguridad informática. Aquí 19/04/2021 


\section{MARCO METODOLÓGICO.}

Este trabajo se desarrolló mediante una metodología es descriptiva y documental, comienza a describir las criptomonas como una opción de inversión, lo que son, su crecimiento o aprecio a lo largo del tiempo, sus riesgos, impacto e incidencia en los mercados, y los beneficios o ventajas se medirán para el medio ambiente, en Caso de ser regulado en Colombia. La Guía de preguntas para la cual es la parte, las monedas virtuales son un fraude, ¿cómo operan, ¿quién puede invertir o controlarlos o regularlos en Colombia? Como investigados, conceptos, variables y tendencias relacionadas con los inversores de moneda virtual y prospectos, lo hará Analizar las posiciones de banca comercial,

De igual manera, el tipo de investigación es descriptivo - explicativo Enfoque Cuantitativo (cuantitativo). De acuerdo a lo anterior es descriptiva, puesto que se involucra la observación y la descripción; es explicativa, porque se busca analizar un fenómeno logrando identificar las causas que ocasionan la problemática y determina efectos.

Este estudio tiene un enfoque cuantitativo pues se van argumentar los resultados de la investigación y se tendrá en cuenta el análisis estadístico, así como también se interpreta La información presentada se realiza de la investigación y, posteriormente, a partir de las fuentes encontradas, dando el uso de diferentes herramientas, como bases de datos, como Benchmark, Vendaval, Libros, Herramientas estadísticas, Bloomberg, páginas estatales de entidades estatales basada en el trabajo en el campo representante de Criptomonedas en Bogotá.

Así mismo según Hurtado (2021) las funciones Cripto-Criptomonedas son sistemas que permiten pagos seguros en línea que están denominados en términos de "tokens" virtuales, que están representados por las entradas de contabilidad del sistema interno.

"Cript" se refiere a los diversos algoritmos de cifrado y técnicas criptográficas que protegen estas entradas, como un cifrado de curvas elípticas, pares públicas y privadas y funciones de Cash.

En apoyo a la presente investigación, se analizarán las estadísticas de volatilidad de las criptomonedas dentro de un período de tiempo específico, y si su especulación está más inclinada al análisis técnico o fundamental (que sucede como cualquier moneda). Se analizará el manejo inicial de monedas virtuales por parte del Banco de la República de Colombia, si la normativa de criptomonedas del país es factible y cuánto ha avanzado el estado en este tema, y tomará en cuenta que, en los negocios internacionales, solo el uso obligatorio de moneda (con valor internacional y la moneda de un país reconocido), pero incluso en algunos países (incluido Colombia), las monedas virtuales se pueden comprar y vender a nivel de inversores privados.

Muestreo y técnicas e instrumentos de recolección de datos

Para este estudio, la adecuada selección de la muestra y para este caso, de acuerdo a la estructura poblacional, se ha elegido el muestreo aleatorio simple. La información presentada se lleva a cabo por la encuesta y el análisis posterior de las fuentes fundadas, dando el uso de diferentes herramientas de oficina, como las bases de datos, de acuerdo a lo anterior, e instrumentos de recolección de la información, Listados de la cinco Cripto monedas más usadas en Colombia. 


\section{RESULTADOS.}

\section{Colombia, Posición Banco de la República frente a las Criptomonedas}

Con respecto a las criptomonedas, el Banco de la República emitió solo advertencias al consumidor sobre el uso y el intercambio de este tipo de monedas virtuales. En este sentido, su primera declaración fue el 1 de abril de 2014 a través de un comunicado de prensa.

En este sentido, según el emisor, debe entenderse que las criptomonedas no han sido reconocidas como una moneda en Colombia. En respuesta a las preocupaciones de muchos sectores de la economía, según lo indicado (Banco de la República, 2017), ni la moneda virtual de Bitcoin o ninguna moneda virtual ha sido reconocida por los legisladores del país o por el Banco Central, porque No corresponde a una moneda o un equivalente activo y sin compromiso es decir sin una autoridad central.

Con estas declaraciones, el Banco de la República deja claro que el régimen de los cambios internacionales no reconoce las criptomonedas, y, por lo tanto, no se pueden usar para transacciones de cambio o divisas. Porque no están activos de que, ya que tienen el respaldo de los bancos centrales de otros países, por otro lado, afecta significativamente las operaciones reguladas del mercado, como las importaciones, las exportaciones, las inversiones extranjeras $\mathrm{y}$ todo lo que concierne al régimen de tipo de cambio colombiano. Como resultado, el Banco de la República citó solo a los ciudadanos alertas sobre el uso de criptomonedas en transacciones en el país, pero también su uso en el extranjero, ya que las monedas virtuales existentes son, según el juicio de (Banco de la República, 2017) Instrumentos que no representan alta liquidez en el mercado.

\section{REGULACIÓN.}

En resumen, el uso de los criptomonedas en Colombia no está autorizado como un mecanismo de transacción y pago a la luz de las disposiciones monetarias actuales emitidas por el Banco de la República, en particular en las disposiciones de la resolución externa n. 8 de 2000 Su Junta de Directores de Directores, en la misma línea, la Superintendencia Financiera de Colombia reitera lo que está indicado por el Banco de la República en diferentes cartas circulares, lo que indica que las entidades que están bajo su vigilancia y control, no están autorizadas a invertir, interferir, custodia con este tipo de herramientas, así como también incluye que no autorice el uso de sus plataformas para operar con criptomonedas.

Por lo tanto, para (Banco de la República, 2017) monedas electrónicas, no constituyen una inversión válida o con la que representan un tipo de apoyo seguro. A pesar de la posición del Banco Central de Colombia, el Senado de la senador Carlos Jiménez López, publicado en su sitio web, PL 028-18, donde según el (Senado de la República, 2018) esta cuenta bajo El título de criptomonedas o Monedas Virtuales, propone regularmente estas monedas en el territorio colombiano, el proyecto inicia los términos conceptualizado para el lenguaje del uso de las criptomonedas, explica el comercio y define esta vigilancia, la inspección y el control serían ejercidos por el Ministerio de Tecnología de la información y de comunicaciones, siguiendo las instrucciones de acuerdo con la Ley 1341 de 2009 , pero el control y la vigilancia que tienden al comercio electrónico, ofrecen y otros serían la industria y el comercio, también determinan las sanciones, por ejemplo, una de ellas indica que aquellos que deseen trabajar con esta herramienta son naturales o legalmente establecidos. La compañía, deben contar una autorización preventiva, también incluye una 
aspiración por separado del 40\%, aproximada en la transacción final con ciertos ajustes, es importante aclarar que al obtener un resultado final a la pérdida no habría impuesto.

De acuerdo con el 23 de octubre de este año, segundo (Senado de la República, 2018) Este proyecto de ley tiene más de 2400 visitas, una cifra que destaca sorprendentemente, en comparación con las visitas de otros proyectos que publican el Senado, por lo que puede alimentar a muchas personas. Interesado en cómo tomar decisiones.

\section{RIESGO.}

Invertir en monedas virtuales indica que existe un riesgo demasiado alto porque son potencialmente vulnerables a las actividades delictivas y las infracciones financieras, como la trata de productos ilegales, la piratería y el robo de identidad (por correo electrónico o portafolios), el manejo y el fraude, facilitan sin licencia. Empresas, capacidad para abrir de forma rápida y anónima para convertir y consolidar el dinero, el financiamiento terrorista y el fraude de sanciones. Un ejemplo de un riesgo de criptomonedas es el siguiente: Según (Holman y Stettner, 2018), las autoridades europeas han identificado una operación de lavado de dinero usando Bitcoin, comprado en una bolsa en Finlandia para transferir el tráfico de tráfico de la droga de España en Colombia y Panamá.

Por lo tanto, se genera la inestabilidad financiera, generando un riesgo a largo plazo porque las transacciones son irreversibles porque no existe una autoridad central para regular y monitorear las transacciones. Tener una visión de nivel macro, como lo hago, según (Abdultef Olalasubomi Abdul LL B., 2017), existen grandes preocupaciones y tendencias en la deflación debido a un tipo de fijación en la provisión de criptomonedas, porque es posible que sea posible que El valor de la moneda nacional de una tienda nacional para disminuir llegan a un auge hasta el punto de reemplazarlo. 
Tabla 1 Descripción y Medidas de Riesgo de Algunas Criptomonedas

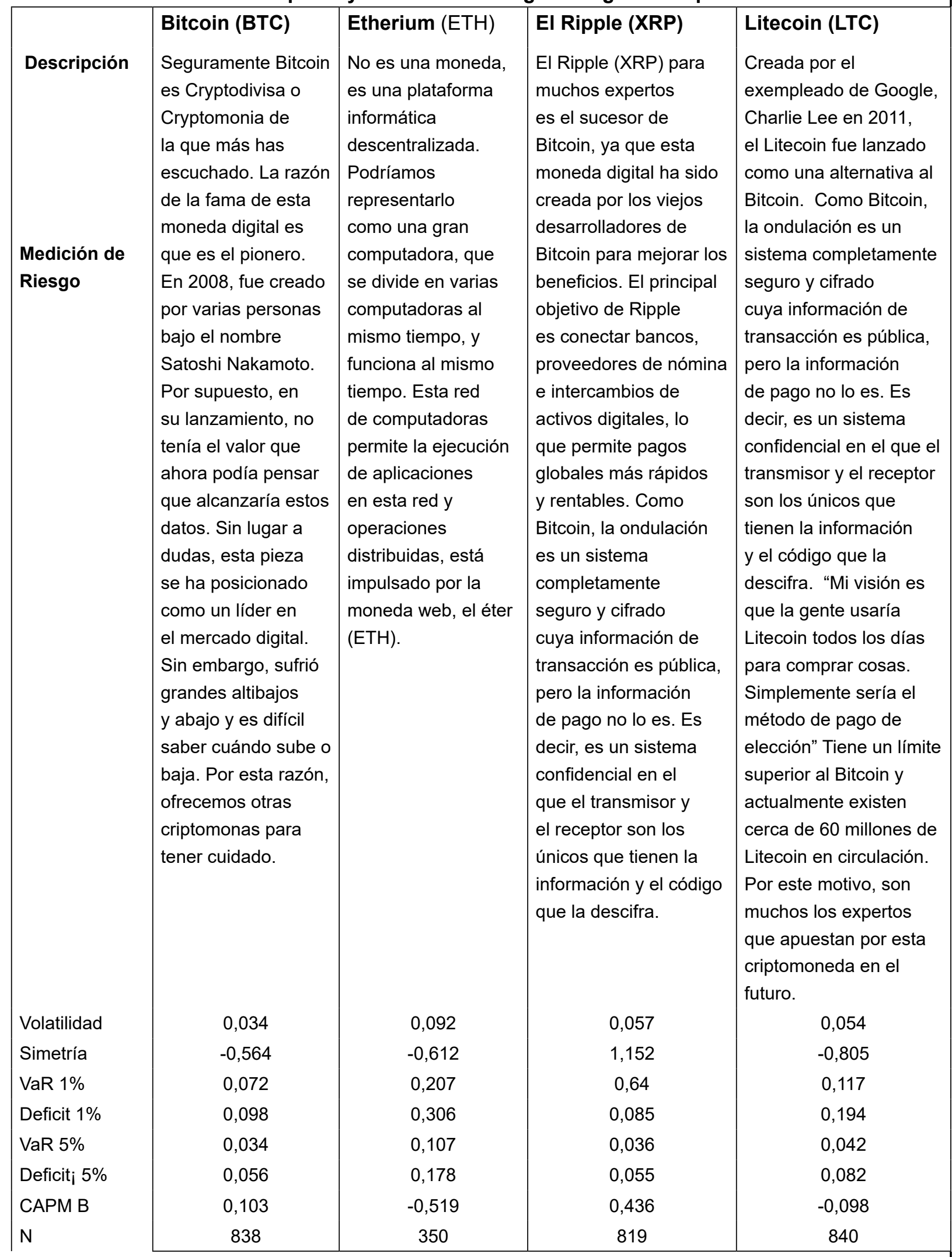




\section{DISCUSIÓN}

La tabla anterior muestra relativamente entre diferentes Criptomoneda algunos parámetros de riesgo, como la volatilidad, la simetría u otros, por ejemplo, el bitcoin es menos volátil

Enaquellos países en los que Criptomoneda no están oficialmente garantizados por su comercialización, y no hay entidad que los regule y en sí, la probabilidad de perder el dinero invertido es muy alto, las personas que realizan esta inversión deben conocer los riesgos intrínsecos. que deben presumir que pueden ganar o perder su dinero porque no hay apoyo de ninguna autoridad monetaria, ya que también es importante aclarar que pueden obtener resultados positivos, ganancias, se debe medir el perfil de riesgo de la inversión, que Una moneda o entidad está regulada no garantiza la cobertura o la confiabilidad de su inversión y devoluciones, se vuelve molesta nombrar el caso de intervención en Colombia, siendo la mejor referencia para un representante o un inversionista de Criptomoneda en comparación con que la regulación sea segura y qué no está regulado no es.

Algunos de los riesgos a los que los operadores Criptomoneda están expuestos a Colombia:

- $\quad$ Las monedas virtuales no tienen ningún tipo de apoyo gubernamental, lo que implica el riesgo en el valor de intercambio de estas monedas virtuales disminuye rápidamente o incluso que vienen al valor de escape.

- Las plataformas virtuales no están reguladas 0 monitoreadas por la Superintendencia Financiera o por la Ley de Colombia, que garantize su operación regularmente, evitando grandes pérdidas de dinero a los inversores. Se puede proporcionar su uso para llevar a cabo actividades ilegales que dañen el desarrollo del país y su economía. - A diferencia de los bancos, los criterios no tienen seguro ni garantía que proteja las inversiones de las personas, en el momento de que cualquier tipo de falla, los inversores pueden permanecer con un saldo negativo en sus cuentas, sin tener una manera de recuperar su inversión.

\section{CONCLUSIONES}

La criptomoneda funciona a través de la tecnología, como cualquier sistema, esta tecnología no es $100 \%$ segura, pero según la criptografía es muy segura.

Las inversiones u operaciones con criptomatones tienen sus riesgos como cualquier inversión, ya que su concepto es parte de una inversión u operación de acciones, no se llama moneda, sino para la Comisión de Futuros de Comercio de EE. UU. (CFTC), es típico como una mercancía, Lo que genera reconocimiento en el mercado de capitales.

- La percepción de la tranquilidad o legalidad de la operación o no con ellos depende del país en el que esté operativo,

- En Colombia, puede operar con ellos como una inversión, las cuestiones del Banco de La República se comunican de manera preventiva, existe una cuenta llamada ley $\mathrm{PL}$ 028-18 en el Senado de la República, con la esperanza de ser revisado, condenado o cancelado, que Determinaría la regulación de las criptomonas en Colombia.

- $\quad$ Cada nación define sus políticas, pero también cada persona define dónde colocar su dinero y tomar la decisión libre de adquirir el nivel de riesgo, se estima que los riesgos son más flotantes de monedas, en lugar de la plataforma, donde la plataforma De este modo, transformando un riesgo de mercado.

- La cartera de Cryptomonas es muy extensa, su líder es Bitcoin, y actualmente muchas empresas aceptan el pago de Bitcoins, 
por ejemplo, por Microsoft, como otros que se desarrollan en esta plataforma, como IBM.

- $\quad$ En Colombia, puede comprar, vender o transferir criptomones usando diferentes aplicaciones o plataformas de Internet, se adquiere una bolsa $y$, si desea monetizar en Colombia, puede usar Cajeros Athena, máquinas que trabajan muy similares a un cajero automático, teniendo como importantes y En cuanta los descuentos por la Comisión, e incluso cuando está en Colombia, no hay regulación, estas máquinas requieren la exploración de la cedula de aquellos que lo usarán, requisitos para registrar la identificación y los movimientos para comprar, venta, retiros de cada individuo.

-

Cryptomoneda representan una opción de inversión descentralizada, así como una opción de pago que acepta para algunas empresas internacionales, funciona bajo una tecnología altamente segura, que podría explotar a muchos inversores a otro campo, sin embargo, se estima que, según las investigaciones realizadas, los criptomones lo harán. No permitir que estén centralizados, tal vez en ese momento pierda todo lo diferencial contra opciones de inversión, como Forex o activo refugio

\section{REFERENCIAS BIBLIOGRÁFICAS}

Abdullateef Olasubomi Abdul (LL.B., B. (2017). Cryptocurrencies in Nigeria: A Legal Analysis. Anti-Money Laundering

BCSM (2021) Miguel Lorente Munilla, Máster en Consultoría de Transformación Digital con Salesforce Las monedas virtuales, o criptodivisas, https://bcsm.es/monedavirtual-miguel lorente/\#: : text=Una $\% 20$ definici\%C3\%B3n\%20simple $\% 20$ sobre $\% 20$ qu $\%$ C3 $\% A 9$, totalmente $\% 20$ implementada\%20es\%20el\%20Bitcoin.

Cámara Colombiana de Comercio Electronico . (05 de 04 de 2018). Cámara Colombiana de Comercio Electronico. Obtenido de Cámara Colombiana de Comercio Electrónico : https://www.ccce.org.co/ noticias/en-el-2017-las-transaccionesdigitales-en-colombia-aumentaron24-con-respecto-al-2016 https:// expeditiorepositorio.utadeo.edu.co/ bitstream/handle/20.500.12010/4746/ tesis $\% 20$ especializacion $\% 20$ criptomonedas-biblioteca. pdf?sequence $=1$ \&isallowed $=y$

Chirino (2021) Regulación y tributación en el mercado de criptoactivos, una perspectiva de derecho comparado http://www. scielo.edu.uy/pdf/rfd/n48/2301-0665-rfd48-e112.pdf

García Díaz, Carlos Mario; Cárdenas Sánchez, Giovanny Alexander; Molina Rodríguez, Carlos Hernando (2011) Análisis de la Intermediación Financiera en el Escenario de las Crisis de los Siglos $\begin{array}{lllll}X X & Y & X X I & \text { https://www.redalyc.org/ }\end{array}$ pdf/4137/413740748010.pdf

Hurtado (2021) Las 10 criptodivisas (o criptomonedas) con más futuro, IEBS Business \& Tech \$ Finanzas Blog https:// www.iebschool.com/blog/criptodivisascriptomonedas-invertir-finanzas/

Lozano (2017) Sistema Financiero Colombiano Miembro de la Redllumno https://digitk.areandina.edu.co/ bitstream/handle/areandina/1502/ Sistema\%20financiero\%20colombiano. pdf? sequence $=1$ \&isAllowed $=y$

Moreno. B, Soto. F, Valencia. N, Sánchez. A (2018) Criptomonedas Como Alternativa de Inversión, Riesgos, Regulación y Posibilidad de Monetización en Colombia

Holman, D., \& Stettner, B. (2018). Anti-Money Laundering Regulation of Cryptocurrency: U.S. and Global Approaches. Allen and Overy LLP. 
Senado de la República. (2018). http://www. senado.gov.co. Obtenido de http://www. senado.gov.co/az-legislativo/proyectosde-ley

Senado de la República. (2018). http://www. senado.gov.co/. Obtenido de http:// www.senado.gov.co/: $\quad$ http://leyes. senado.gov.co/proyectos/images/ documentos/Textos\%20Radicados/ proye Superintendencia Financiera de Colombia. (22 de 06 de 2017). Superintendencia Financiera de Colombia-Comunicado de Prensa. Obtenido de https://www.superfinanciera. gov.co/publicacion/monedas-virtuales-ocriptomonedas-10090492

Tradingview. (28 de 05 de 2018). Tradingview. Obtenido de https://es.tradingview.com/ markets/cryptocurrencies/prices-all/ ctos $\% 20 \mathrm{de} \% 20$ ley $/ 2018 \% 20-\% 202019 /$ PL\%20028-18\%20Criptomonedas.pdf 